\title{
A Study of Integrated Driver fatigue Judging Algorithm Based on Principal Component Analysis
}

\author{
Zhipeng $M a^{1, *}$, Shuwan $\mathrm{Yao}^{2}$, and Junxian $\mathrm{Li}^{1}$ \\ ${ }^{1}$ College of Automotive Engineering, Jilin University, 130022 Changchun, China \\ ${ }^{2}$ College of Mechanical Science and Engineering, Jilin University, 130022 Changchun, China
}

\begin{abstract}
As the number of vehicles increases, the probability of fatigue driving rises accordingly, resulting in a boost in the accident rate. Based on principal component analysis, the paper presents a fatigue monitoring algorithm that integrates with facial, EEG, EMG and ECG. First of all, based on the edge detection method, the eyes are positioned and then it has an assessment through the Perclos fatigue. EEG, EMG, ECG indicators evaluate the fatigue with the corresponding fatigue characteristics. Subsequently, the related values of the above four indexes are normalized, and the principal component analysis method (PCA) is utilized to reduce the dimension and merge to get the comprehensive fatigue eigenvalue, and then we get the fatigue level referring to the fatigue level standard. Analyzing the measured data, the algorithm accuracy is $76 \%$.
\end{abstract}

\section{Introduction}

With the increase of motor vehicle ownership, road traffic mileage and the fatigue index of the modern people, the phenomenon of fatigue driving intensified. Fatigue driving accident rate is high, resulting in serious consequences and huge losses [1]. Fatigue driving monitoring technology is highly valued by all the countries due to its broad development prospects in the prevention of traffic accidents. There has been a great deal of research on fatigue driving both in China and abroad.

Carnegie Mellon proposed PERCLOS [2] and demonstrated that the percentage of total eye closure over $80 \%$ of total time per unit of time can bestly indicate driving fatigue. In 1993, the Swartz Center for Computational Neurosciences at the University of California, San Diego, began a study of fatigue monitoring through electroencephalography (EEG), mapping out the correspondence between fatigue and EEG spectral features [3]. However, Single-index analysis is greatly affected by individual differences and contingencies, so multi-feature fusion is a research trend. In 2004, the EU puts forward the AWAKE Plan [4], developed a set of driving fatigue monitoring and early warning system that integrates with various behaviors of drivers in driving process. The system analyzes these behavioral parameters to determine whether it is necessary to provide drivers with a warning reminder. Rajiv, Ranjan, Singh, et al. [5] combined the physiological indexes of skin electricity with light volume pulse, using neural network to discriminate fatigue driving, where the recognition rate is high.

\section{Establishment of Subsystem Analysis Model}

\subsection{Analysis model based on eye movement}

\subsubsection{Overview of eye location algorithm}

Eye location algorithm can be divided into activeinfrared-based eye location algorithm and image-based passive eye location algorithm. Eye location algorithm based on active infrared use the basic principle of "redeye" in flash photography and it requires the acquisition of face images using a camera equipped with an infrared light source. Image-based passive eye location algorithms mainly include projection function, Hough transform, template matching statistical methods and so on. [6]

Active algorithm is relatively simple, which can get relatively high eye position and tracking accuracy. Nevertheless, the accuracy of the passive algorithm can be affected, when closing eyes, shutting the light, etc. But it is less affected by the outside surroundings, so it has become the mainstream of current research.

\subsubsection{Establishment of eye-based fatigue assessment model}

In the paper, an eye location algorithm based on edge detection method is put forward and Perclos fatigue assessment is possessed. Each frame of image is evenly divided into $20 * 20$ rectangles for edge extraction. It is

\footnotetext{
Corresponding author:mzp1997@sina.com
} 
programmed through MATLAB, and extract 6 frames per second are to process the true time video. Perclos fatigue evaluation threshold is 0.7 . Fatigue evaluation algorithm is as shown in Fig. 1. The eye location algorithm is as shown in Fig. 2.

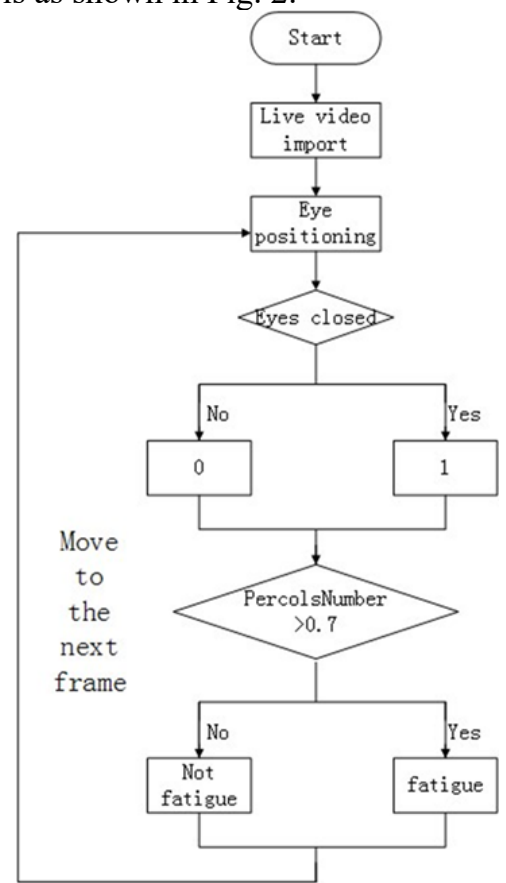

Fig. 1. Fatigue Evaluation Algorithm

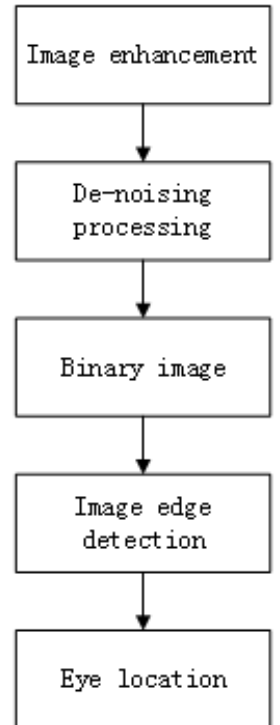

Fig. 2. Eye Location Algorithm

Among them, linear transformation is used for image enhancement, Gaussian Low Pass Filter is used for denoising processing and Canny operator is used for image edge detection. The results are shown in Fig. 3.

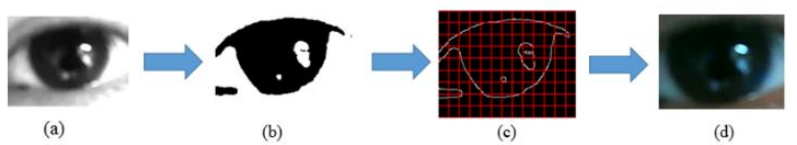

Fig. 3. Eyeball Positioning Algorithm Renderings

Fig. 3 (a) is an enhanced image, and Fig. 3 (b) is a binarized image. Fig. 3 (c) is for the regional segmentation and edge detection of the image, Fig. 3 (d) is the cut image after location.

\subsection{Analysis model based on the physiological information fatigue}

\subsubsection{Physiological information analysis model overview}

Physiological indicators associated with fatigue include EEG, ECG, EMG and so on. In 1993, the Swartz Center for Computational Neurosciences at the University of California, San Diego, began a study of fatigue monitoring by electroencephalography (EEG) and found the correspondence between fatigue and EEG spectral features [3]. Wilson et al. [7] found that heart rate variability is closely related to the degree of fatigue and can reflect the psychological and physiological load of the human body. A Japanese company [8] perceived the pulse of a driver's hand while holding the steering wheel by installing a sensor on the steering wheel and analyzed whether the driver was in a driving fatigue state by using a corresponding algorithm.

In this paper, three indicators of EEG, ECG and EMG were studied and their fatigue monitoring models were analyzed.

\subsubsection{Establishment of EEG-based fatigue assessment model}

In this paper, a brainwave sensor named Neurosky makes the acquisition of brain wave data. According to the serial protocol, brainwave module data frames sent 513 packets per second. Through the preprocessing of the analysis software, a variety of brain wave data were obtained, in which there were $\theta, \alpha$ and $\beta$ waves with large fatigue correlation, and the package was analyzed by the feature of $(\alpha+\theta) /(\alpha+\beta)$, and then through normalization and testing, the range of eigenvalues is 0 to 100 , where 0 is awake, 100 is fatigue.

\subsubsection{Establishment of EMG-based fatigue assessment model}

In this paper, an EMG sensor named myoware, which monitors the surface EMG (sEMG) frequency from the neck and back muscles, makes data acquisition and signal spectrum analysis. MPF is a critical eigenvalue of the drowsy-driving analysis. [11] The function is shown in (1).

$$
f_{M P F}=\frac{\int_{0}^{f_{s} / 2} f P(f) d f}{\int_{0}^{f_{s} / 2} P(f) d f}
$$

Where $f_{M P F}(\mathrm{~Hz})$ is the number of MPF; $f(\mathrm{~Hz})$ is the EMG signal frequency; $f_{s}(\mathrm{~Hz})$ is the sample frequency; $P(f)\left(\mu \mathrm{V}^{2} / \mathrm{Hz}\right)$ is for the power spectral density (PSD) function.

From experiments, the degree of fatigue deepens with time. As the degree of fatigue increases, MPF 
generally declines. If MPF declines to $120 \mathrm{~Hz}$, it could be assessed as a drowsy situation.

\subsubsection{Establishment of ECG-based fatigue assessment model}

The paper is based on an ECG sensor named Pulsesensor, which is put on the neck, to accomplish data acquisition and analysis. The weak signal magnifies by 330 times, the data are recorded every $20 \mathrm{~ms}$. Power is the key eigenvalue of the drowsy-driving analysis. Take the data from every $500 \mathrm{~ms}$ to integrate, the corresponding power value is available. According to the experiments, the degree of fatigue deepens with time. When the power value is less than $320 \mu \mathrm{w}$, it can be determined that the driver is at a drowsy state.

\section{Research on Multi-Feature Fusion Algorithm}

\subsection{Multi-feature fusion of the general algorithm}

In order to improve the generalization ability and robustness of the fatigue assessment algorithm, it is necessary to conduct a comprehensive evaluation of multiple indicators. Awake project uses a variety of means of detection signal fusion, including steering wheel grip change detection, steering wheel angle change detection, driver's eye condition detection, and lane line tracking detection. According to the test results, the driver's current situation can be classified into three categories named awake, light fatigue and deep fatigue [8]. Rajiv Ranjan Singh et al researched the skin electricity and light volume pulse physiological indicators and used neural network to assess the driving fatigue, which achieved a good recognition rate of $89.23 \%$ [5]. Wang Ning [9] adopted the method of support vector machine (SVM) to build the fatigue situation identification classifier for high-dimensional matrix and analyzed the test results, which also achieved a good efficiency.

\subsection{Principal Component Analysis}

In the paper, the principal component analysis method (PCA) is utilized to make a comprehensive analysis of the four indicators, through principal component analysis, we can effectively reduce the data dimension, extract valid information, so as to remove redundant information and improve the efficiency of the operation. Principal component analysis is a widely used method of dimensionality reduction in fields such as image analysis and data mining. Its main idea is to convert multiple variables in a multi-dimensional eigenvector into a smaller number of principal components which are linear combinations of the initial features, while satisfying the condition that the data variables are linearly independent of each other. (That is, the $n$-dimensional feature is mapped to the $\mathrm{k}$-dimension, and $\mathrm{k}<\mathrm{n}$ ) to reconstruct a brand new k-dimensional orthogonal feature so as to achieve the dimension reduction [9].

The data measured by the four indexes are normalized, and a series of analysis and processing are conducted. The principal component is selected as a new index of evaluation when the cumulative contribution rate is $90 \%$, and the algorithm is shown in Fig. 4.

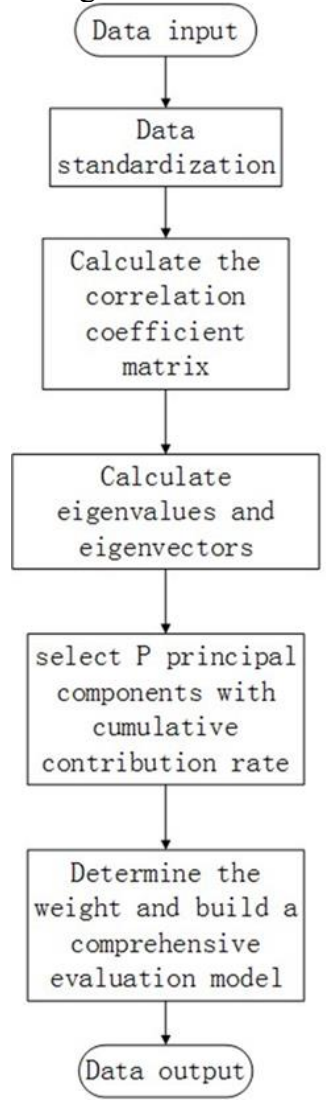

Fig. 4. PCA Algorithm Flow Chart

Merits of the principal component analysis are that the elimination of the correlation among the assessment criteria can reduce the workload of index selection. When there are many rating indicators, we can use a small number of comprehensive indexes instead of the original parameters to analyze while retaining the vast majority of messages.

\section{Result of Application and Discussion}

50 drivers were selected as the experimental samples. 30 of them were males and 20 were females, aged 20-50 years old. The Perclos value, EEG, EMG and ECG data of each group were collected and then the data are analysed.

The miniature camera is used for eye image acquisition, image data input processor for eye location and further processing to obtain Perclos data. The accuracy of eye location was $72 \%$.

The collected Perclos value, EEG data, EMG data, ECG data were normalized, the result can be represented among the value of 0 to 1 , where 0 means the most sober and 1 means the most tired.

According to Fig. 4, the algorithm is programmed with MATLAB. When the cumulative contribution rate 
is $90 \%$, a total of three principal components were obtained, the PCA vector F is:

$$
\mathrm{F}=\left[\begin{array}{rrrr}
-0.6182 & 0.3841 & 0.1298 & 0.5045 \\
-0.2128 & -0.4402 & -0.6778 & 0.3563 \\
0.0517 & -0.3388 & 0.1391 & -0.3277
\end{array}\right]\left[\begin{array}{l}
X_{1} \\
X_{2} \\
X_{3} \\
X_{4}
\end{array}\right]
$$

Where $\mathrm{X} 1 \sim \mathrm{X} 4$ are eye, EEG, EMG and ECG data.

The weight can be normalized by the contribution rate, then the weight matrix $\mathbf{W}$ can be represented:

$$
\mathbf{W}=\left[\begin{array}{llll}
0.3566 & 0.2714 & 0.1999 & 0.1721
\end{array}\right]
$$

Fatigue score vector $\mathbf{S}$ is as follows:

$$
\mathbf{S}=\mathbf{W F}
$$

After calculating the comprehensive fatigue eigenvalue, the fatigue scale is determined. 0 to 0.2 means awake, 0.2 to 0.5 means light fatigue, 0.5 to 0.7 means moderate fatigue, 0.7 to 1.0 means deep fatigue, and different states correspond to different alarm modes. The accuracy of the model tested through the samples is $76 \%$.

\section{CONCLUSION}

Driver fatigue is caused by a very complex mechanism, because there are many factors interacting with fatigue [1]. Driver fatigue is accumulated over time, so our research goal is to establish a fatigue test model to monitor the drivers in real time.

In this paper, the way of edge detection is utilized to locate the eyeball and the perclos method is utilized to evaluate the fatigue. Then it comprehensively combined the three physiological indexes such as EEG, EMG, ECG and facial indexes, and made the comprehensive fatigue evaluation based on PCA. The feasibility and validity of the judgment are good.

Nowadays, driving fatigue evaluation algorithm and system needs more in-depth research to enhance the robustness and generalization capability of the system to reduce the incidence of accidents caused by fatigue [10].

\section{References}

1. Rongrong Fu, Hong Wang, Wenbo Zhao. Dynamic driver fatigue detection using hidden Markov model in real driving condition. Expert Systems with Applications, 63 (2016) 397-411.

2. DINGES D F, MALLIS M, MAISLING, etal. Evaluation of techniques for ocular measurement as an index of fatigue and the basis for alertness management, National Highway Traffic Safety Administration, USA, 1998.

3. T.P. JUNG, MAKEIG S, SENSMO M, etal. Estimating Alertness from the EEG Power Spectrum. IEEE Transactions on Biomedical Engineering, 1997, 44(1):60-69.

4. BOVERIS S. Driver fatigue monitoring technologies and future ideas, Proceedings of
AWAKE Road Safety Workshop. Balocco, Italy: Elsevier Science Ltd, 2004.

5. S. Miloevic. Driver's fatigue studies. Economics, 1997, 40(3):381-38.

6. He Qiwen. Research on driver fatigue detection based on multi-feature integration. Changsha: Central South University of Forestry and Technology, 2009.

7. G.F.Wilson, R.D.Donnell. Measurement of operator workload with the neuropsychological workload test battery. Advances in Psychology, 1988, (52):63-100.

8. LIN C, K0 L, CHUNG I, et al. Adaptive EEG-Based Alertness Estimation System by Using ICA-Based Fuzzy Neural Networks. IEEE Transactions on Circuits and Systems-I, 2006, 53(11):2469-2476.

9. Wang Ning. Study of fatigue state recognition based on multiple information fusion. Jinan: Shandong University, 2016.

10. Mengzhu Guo, Shiwu Li, Linhong Wang, Meng Chai, Facheng Chen and Yunong Wei. Research on the Relationship between Reaction Ability and Mental State for Online Assessment of Driving Fatigue. Public Health, 2016, 13, 1174.

11. Du Yunxiao, Wang Shuyi, Ndaro Nyakuru Zaphlene, ZUO Yan. Comparative study on evaluation algorithms for neck muscle fatigue based on surface electromyography signal. Journal of Biomedical Engineering. 2018, Vol. 35, No. 1: 31-37 